\title{
New Public-Key Schemes Based on Elliptic Curves over the Ring $\mathbf{Z}_{n}$
}

\author{
Kenji Koyama* \\ Ucli M. Maurer ${ }^{\dagger}$ \\ Tatsualii Okamoto ${ }^{\ddagger}$ \\ Scott A. Vanstone $e^{\S}$
}

\begin{abstract}
Three new trapdoor one-way functions are proposed that are based on elliptic curves over the ring $Z_{n}$. The first class of functions is a naive construction, which can be used only in a digital signature scheme, and not in a public-key cryptosystem. The second, preferred class of function, does not suffer from this problem and can be used for the same applications as the RSA trapdoor one-way function, including zero-knowledge identification protocols. The third class of functions has similar properties to the Rabin trapdoor one-way functions. Although the security of these proposed schemes is based on the difficulty of factoring $n$, like the RSA and Rabin schemes, these schemes seem to be more secure than those schemes from the vicwpoint of attacks without factoring such as low multiplier attacks. The new schemes are somewhat less efficient than the RSA and Rabin schemes.
\end{abstract}

\section{Introduction}

In their seminal 1976 paper [3], Diffe and Hellman introduced the concept of a trapdoor one-way function (TOF). A TOF is a function that is easy to evaluate but infcasible to invert, unless a secret trapdoor is known, in which case the inversion is also easy. Although no realisation of a TOF was proposed in [3], Diffie and Hellman observed that such a function would allow the construction of digital signature schemes and public-key cryptosystems, two concepts that they introduced.

The first implementation of a TOF was proposed by Rivest, Shamir and Adleman in 1978 [21]. Its security relies on the difficulty of factoring a composite number $n$. Some other implementations $[20,4]$ of TOFs have been proposed based on the difficulty of factoring and discrete logarithms. From another direction, one of the recent topics in the ficld of elliptic curves is their applicability to cryptography. The points of an elliptic curve $E$ over a finite ficld form an abclian group, and hence the group $E$ can be used to implement analogs of the Diffie-Hellman key exchange scheme and the ElGamal public key cryptosystem, as explained in [9]. The security of these analogous systems rests on the difficulty of the discrete logarithm problem on an elliptic curve.

"NTT Laboratories, Sanpeidani, Inuidani, Seikacho, Kyoto, 619-02, Japan

IPrinceton University, Princeton, NJ 08544, USA; Supported by Omnisec AG, Switzerland

'NTT Laboratories, Yokosuka-shi, Kanagawa 238-03, Japan

$\S$ University of Waterloo, Ontario, N2L 3G1, Canada

J. Feigenbaum (Ed.): Advances in Cryptology - CRYPTO '91, LNCS 576, pp. 252-266, 1992.

(C) Springer-Verlag Berlin Heidelberg 1992 
In this paper, we propose new TOFs (or public-key cryptographic schemes) based on elliptic curves over a ring $\mathrm{Z}_{n}$. The security of these TOFs depends on the difficulty of factoring $n$. Although these schemes are less efficient than the PSA and Rabin schemes, our schemes seem to be more secure from the viewpoint of some attacks that do not use factoring such as low multiplier attacks. In this case, even when the RSA system can be broken without factoring the modulus, our schemes seem to remain secure.

We begin with a brief review of the basic definitions and facts about elliptic curves over a finite field. In Section 3, we show some properties of elliptic curves over a ring, which are used in the succeeding sections. Section 4 proposes a naive construction of the TOF (Type 0 scheme) based on elliptic curves over a ring, but which can be used only in a digital signature scheme, and not in a public-key cryptosystem. In Sections 5 and 6 , we propose the Type 1 and Typc 2 schemes respectively based on the elliptic curve over a ring, and discuss their properties. Section 7 discusses the sccurity of the proposed schemes, and Section 8 discusses their performance.

\section{Elliptic Curves over a Finite Field}

Let $K$ be a field of characteristic $\neq 2,3$, and let $a, b \in K$ be two parameters satisfying $4 a^{3}+27 b^{2} \neq 0$. An elliptic curve over $K$ with parameters $a$ and $b$ is defined as the set of points $(x, y)$ with $x, y \in K$ satisfying the equation

$$
y^{2}=x^{3}+a x+b
$$

together with a special element denoted $\mathcal{O}$ and called the point at infinity. We will mainly be interested in elliptic curves over the finite field $F_{p}$ with $p$ elements, for some prime $p$. Such a curve will be denoted $E_{p}(a, b)$. What makes elliptic curves interesting in cryptography is the fact that an addition operation on the points of an elliptic curve can be defined that makes it into an abelian group. This addition operation, which has but its name in common with the ordinary addition of integers, is described in the following.

Let $E$ be an elliptic curve, and let $P$ and $Q$ be two points on $E$. The point $P+Q$ is defined according to the following rules. If $P=\mathcal{O}$, then $-P=\mathcal{O}$, and $P+Q=Q$ (i.e., $\mathcal{O}$ is the neutral element of $E)$. Let $P=\left(x_{1}, y_{1}\right)$ and $Q=\left(x_{2}, y_{2}\right)$. If $x_{1}=x_{2}$ and $y_{1}=-y_{2}$, then $P+Q=\mathcal{O}$ (i.e., the negative of the point $(x, y)$ is the point $(x,-y)$ ). In all other cases the coordinates of $P+Q=\left(x_{3}, y_{3}\right)$ are computed as follows. Let $\lambda$ be defined as

$$
\lambda= \begin{cases}\frac{y_{2}-y_{1}}{x_{2}-x_{1}} & \text { if } x_{1} \neq x_{2} \\ \frac{3 x_{1}^{2}+a}{2 y_{1}} & \text { if } x_{1}=x_{2} .\end{cases}
$$

(When $P+Q \neq \mathcal{O}$, then the denominator is always non-zero and thus the quotient is defined.) The resulting point $P+Q=\left(x_{3}, y_{3}\right)$ is defined by

$$
\begin{aligned}
& x_{3}=\lambda^{2}-x_{1}-x_{2} \\
& y_{3}=\lambda\left(x_{1}-x_{3}\right)-y_{1} .
\end{aligned}
$$

Clearly, the first equation is equivalent to $x_{3}=\lambda^{2}-2 x_{1}$ when $P=Q$. All computations are in the field over which $E$ is defined. In particular, when the field is $F_{p}$, all computations are modulo $p$. 
Let $\# E_{p}(a, b)$ denote the order (i.e., the number of points) of the elliptic curve $E_{p}(a, b)$. It is well-known that \# $E_{p}(a, b)=p+1+t$ where $|t| \leq 2 \sqrt{p}$ for every elliptic curve over $\mathbf{F}_{p}$. Every value of $t$ within the given bounds is taken for some pair $(a, b)$, but this fact will not be used in this paper. There exists a polynomial-time algorithm due to Schoof [22] for computing the order of an elliptic curve, but this algorithm is quite impractical for large $p$. It is known that $E_{p}(a, b)$ is either cyclic or the product of two cyclic groups. In the latter case, $E_{p}(a, b) \cong \mathrm{Z}_{N_{1}} \times \mathrm{Z}_{N_{2}}$ where $N_{1} \cdot N_{2}=\# E_{p}(a, b)$, where $N_{2}$ divides $N_{1}$ and where $N_{2}$ also divides $p-1$. We refer to [9] for a more detailed introduction to elliptic curves, and to [8] for some further cryptographically useful properties of elliptic curves.

For some special classes of elliptic curves the order and group structure is easily determined. The following well known two lemmas illustrate this point.

Lemma 1. Let $p$ be an odd prime satisfying $p \equiv 2(\bmod 3)$. Then, for $0<6<p$, $E_{p}(0, b)$ is a cyclic group of order

$$
\# E_{p}(0, b)=p+1 .
$$

Proof. We first prove that $\# E_{p}(0, b)=p+1$. When $p \equiv 2(\bmod 3)$ then the mapping $x \mapsto x^{3}$ is a permutation on $\mathrm{F}_{p}$. Hence for every $b$ there are exactly $(p-1) / 2$ numbers $x \in \mathbf{F}_{p}$ for which $x^{3}+b$ is a quadratic residue, and for each such $x$ there are two points on $E_{p}(0, b)$, viz., the points $\left(x, \pm \sqrt{x^{3}+b}\right)$. Together with the points $(\sqrt[3]{-b}, 0)$ and $\mathcal{O}$ there are $p+1$ points on $E_{p}(0, b)$. To prove that $E_{p}(0, b)$ is cyclic (see also [8]), suppose it is not. Then $E_{p}(0, b) \cong \mathrm{Z}_{N_{1}} \times \mathrm{Z}_{N_{2}}$ where $N_{1} N_{2}=p+1$ and $N_{2}$ divides $p-1$. Hence $N_{2}=2$ and $N_{1}$ is even. Then the group $Z_{N_{1}} \times Z_{2}$ must have four elements $P$ for which $-P=P$. However, there are exactly two points, $P=\mathcal{O}$ and $(\sqrt[3]{(-b)}, 0)$ for which $-P=P$, since the only points $P$ on $E_{p}(0, b)$ for which $-P=P$ are the points $(x, y)$ with $y=0$. This contradiction implies $E_{p}(0, b)$ is cyclic.

Lemma 2. Let $p$ be a prime satisfying $p \equiv 3(\bmod 4)$. Then, for $0<a<p$, we have

$$
\# E_{p}(a, 0)=p+1 \text {. }
$$

Moneover, $E_{p}(a, 0)$ is cyclic if $a$ is a quadratic residue modulo $p$ and $E_{p}(a, 0) \cong \mathbf{Z}_{(p+1) / 2} \times$ $\mathrm{Z}_{2}$ otherwise.

Proof. Let $f(x)=x^{3}+a x . f(x)$ is an odd function, i.e., $f(-x)=-f(x)$. The condition $p \equiv 3(\bmod 4)$ implies that for every $s \in Z_{p}^{*}$, exactly one of the two numbers $s$ or $-s$ is a quadratic residue modulo $p$. This follows from the fact that $(p-1) / 2$ is odd and thus -1 is a quadratic non-residue modulo $p$. Consider the $(p-1) / 2$ pairs $[x,-x]$ for $0<x \leq(p-1) / 2$. For every such pair, either $f(x)=f(-x)=0$ or $f(x)$ is a quadratic residue or $f(-x)$ is a quadratic residue. In either of the three cases, there exist 2 point on $E_{p}(a, b)$ associated with the pair $[x,-x]$, viz., $( \pm x, 0),(x, \pm \sqrt{f(x)})$ or $(-x, \pm \sqrt{-f(x)})$, respectively. Together with $(0,0)$ and $\mathcal{O}$ the total number of points on $E_{p}(a, b)$ is $p+1$. The proof of the last claim is similar to the proof given for Lemma 1.

\section{Elliptic Curves over a Ring}

We now consider elliptic curves over the ring $Z_{n}$, where $n$ is an odd composite squarefrce integer. (An alternative notation for $Z_{n}$ used in the literature is $Z / n Z$.) Similar to the 
definition of $E_{p}(a, b)$, an elliptic curve $E_{n}(a, b)$ can be defined as the set of pairs $(x, y) \in \mathbf{Z}_{n}^{2}$ satisfying $y^{2} \equiv x^{3}+a x+b(\bmod n)$, together with a point $\mathcal{O}$ at infinity. An addition operation on $E_{n}(a, b)$ can be defined in the same way as the addition operation on $E_{p}(a, b)$, simply by replacing computations in $F_{p}$ by computations in $Z_{n}$. However, two problems occur. The first problem is that because the computation of $\lambda$ requires a division which in a ring is defined only when the divisor is a unit, the addition operation on $E_{n}(a, b)$ is not always defined. The second problem, which is related to the first is that $E_{n}(a, b)$ is not a group. It would therefore seem impossible to base a cryptographic system on $E_{n}(a, b)$. In the following we present a natural solution to these problems.

For the sake of simplicity, let $n=p q$ in the sequel be the product of only two primes as in the RSA system. Moreover, the addition operation on $E_{n}(a, b)$ described above, whenever it is defined, is equivalent to the (componentwise defined) group operation on $E_{p}(a, b) \times E_{q}(a, b)$. By the Chinesc Remainder Theorem, every element $c$ of $\mathrm{Z}_{n}$ can be represented uniquely as a pair $\left[c_{p}, c_{q}\right]$ where $c_{p} \in Z_{p}$ and $c_{q} \in Z_{q}$. Thus every point $P=(x, y)$ on $E_{n}(a, b)$ can be represented uniquely as a pair $\left[P_{p}, P_{q}\right]=\left[\left(x_{p}, y_{p}\right),\left(x_{q}, y_{q}\right)\right]$ where $P_{p} \in E_{p}(a, b)$ and $P_{q} \in E_{q}(a, b)$, with the convention that $\mathcal{O}$ is represented by $\left[\mathcal{O}_{p}, \mathcal{O}_{q}\right]$, where $\mathcal{O}_{p}$ and $\mathcal{O}_{q}$ are the points at infinity on $E_{p}(a, b)$ and $E_{q}(a, b)$, respectively. By this mapping, all elements of $E_{p}(a, b) \times E_{q}(a, b)$ are exhausted except the pairs of points $\left[P_{p}, P_{q}\right]$ for which exactly one of the points $P_{p}$ and $P_{q}$ is the point at infinity. Note that the addition operation on $E_{n}(a, b)$ described above is undefined if and only if the resulting point, when interpreted as an element of $E_{p}(a, b) \times E_{q}(a, b)$, is one of these special points.

It is important to note that when all prime factors of $n$ are large, it is extremely unlikely that the sum of two points on $E_{n}(a, b)$ is undefined. In fact, if the probability of the addition operation being undefined were non-negligible, then the very execution of a computation on $E_{n}(a, b)$ would be a feasible factoring algorithm, which is assumed not to exist. Therefore, the first problem will cause no difficulties in practice.

The second problem, that $E_{n}(a, b)$ is not a group, can be solved by the following lemma. That is, although we cannot use the properties of a finite group directly, we can use a property of $E_{n}(a, b)$ which is similar to that of a finite group. The following lemma can be easily obtained from the Chinese Remainder Theorem.

Lemma 3. Let $E_{n}(a, b)$ be an elliptic curve such that $\operatorname{gcd}\left(4 a^{3}+27 b^{2}, n\right)=1$ and $n=p q$ $\left(p, q\right.$ : prime). Let $N_{n}$ be $\operatorname{lcm}\left(\# E_{p}(a, b), \# E_{q}(a, b)\right)$. Then, for any $P \in E_{n}(a, b)$, and any integer $k$,

$$
\left(k \cdot N_{n}+1\right) \cdot P=P \text { over } E_{n}(a, b) \text {. }
$$

We should note that it is possible to define an elliptic curve over a ring so that the resulting structure is a group. For our purposes, this is unnecessary.

\section{Naive Construction of TOF Based on Elliptic Curves over a Ring}

In this section, we show a naive construction of TOFs (Type 0 scheme) which are based on elliptic curves over a ring. These TOFs can be used only in a digital signature scheme, and not in a public-key cryptosystem. The shortcomings of the TOFs of this section are elliminated in the Type 1 and 2 schemes shown in following sections.

A digital signature scheme based on $E_{n}(a, b)$ can be set up as follows. The signer Alice chooses two primes $p$ and $q$ (or, more generally, a set of two or more distinct primes) and two parameters $a$ and $b$ satisfying $\operatorname{gcd}\left(4 a^{3}+27 b^{2}, n\right)=1$, where $n=p q$. She 
then computes the orders of the elliptic curves $E_{p}(a, b)$ and $E_{q}(a, b)$ (for example using Schoof's algorithm [22]), chooses a public encryption multiple $e$ relalively prime to both $\# E_{p}(a, b)$ and \# $E_{q}(a, b)$, and computes the secret decryption multiple $d$ according to

$$
d \equiv e^{-1}\left(\bmod \operatorname{lcm}\left(\# E_{p}(a, b), \# E_{q}(a, b)\right)\right) .
$$

Alice releases as public parameters $n, a, b$ and $e$. When she later wants to sign a message $M$ she associates a point $P=(x, y) \in E_{n}(a, b)$ with $M$ in a publicly-known way (see below) and computes the point $Q=(s, t)$ on $E_{n}(a, b)$ according to

$$
Q=(s, t)=d \cdot P \text {. }
$$

The signature for the message $M$ is the pair $(s, t)$, which can be checked by computing

$$
P=(x, y)=e \cdot Q
$$

on $E_{n}(a, b)$ and extracting the message $M$ from $(x, y)$ (because $(e d) \cdot P=P$ from Lemma 3).

Here, given a message $M$, a point $(x, y)$ on $E_{n}(a, b)$ can efficiently be associated with $M . M$ is first padded with sufficient redundancy, for instance by appending zero's to $M$, resulting in $M^{\prime}$. is defined as the smallest integer greater or equal to $M^{\prime}$ such that $x^{3}+a x+b$ is a quadratic residue modulo $n$, and $y$ is defined as one of the square roots modulo $n$ of this number.

The shortcomings of this scheme are as follows:

(1) Schoof's algorithm [22] to compute \# $E_{p}(a, b)$ and \# $E_{q}(a, b)$ is infeasible for large $p$.

(2) The signature is roughly twice as long as the original message $M$.

(3) This scheme cannot be used for a public-key cryptosystem, since knowledge of the trapdoor is required to create a point on $E_{n}(a, b)$, which corresponds to a plaintext.

This scheme may be advantageous in some circumstances. It does allow digital signature without the possibility of encryption.

\section{Basic TOF Based on Elliptic Curves over a Ring}

In this section, we propose a new TOF (Type 1 scheme) that is based on elliptic curves over a ring. It overcomes the three shortcomings of the Type 0 scheme. For simplicity, we show a protocol for a public-key cryptosystem based on elliptic curves as described in Lemma 1 . We can easily construct a public-key cryptosystem in the case of Lemma 2, and digital signature schemes, although we omit a description.

Step 0 (Key Generation) User U chooses large primes $p$ and $q$ such that

$$
p \equiv q \equiv 2(\bmod 3) \text {. }
$$

U computes the product $n=p q$, and $N_{n}=\operatorname{lcm}\left(\# E_{p}(0, b), \# E_{q}(0, b)\right)=\operatorname{lcm}(p+$ $1, q+1)$.

$\mathrm{U}$ chooses an integer $e$ which is coprime to $N_{n}$, and computes an integer $d$ such that

$$
e d \equiv 1\left(\bmod N_{n}\right) \text {. }
$$

Summarizing, U's secret key is $d,\left(p, q, \# E_{p}(0, b), \# E_{q}(0, b), N_{n}\right)$, and U's public key is $n$, e. 
Step 1 (Encryption) A plaintext $M=\left(m_{x}, m_{y}\right)$ is an integer pair, where $m_{x} \in Z_{n}$, $m_{y} \in \mathrm{Z}_{n}$. Let $M=\left(m_{x}, m_{y}\right)$ be a point on the elliptic curve $E_{n}(0, b)$, where $b$ is determined by $m_{x}$ and $m_{y}$.

Sender A encrypts the point $M$ by encryption function $\mathrm{E}(\cdot)$ with the receiver's public key $e$ and $n$ as

$$
C=\mathbf{E}(M)=e \cdot M \text { over } E_{n}(0, b),
$$

and sends a ciphertext pair $C=\left(c_{x}, c_{y}\right)$ to a receiver $\mathrm{B}$.

Step 2 (Decryption) Receiver B decrypts a point $C$ by decryption function $\mathbf{D}(\cdot)$ with his secret key $d$ and public key $n$ as

$$
M=\mathrm{D}(C)=d \cdot C \text { over } E_{n}(0, b) .
$$

\section{[Notes]}

1. In the case of Lemma 1, the minimum possible value of $e$ is 5 because $2 \mid N_{n}$ and $3 \mid N_{n}$. In the case of Lemma 2 , the minimum possible value of $e$ is 3 because $2 \mid N_{n}$.

2. For elliptic curves, the addition formula is independent of $a$ and $b$, and the doubling formula is independent of $b$. Thus, the above protocol does not require computation of the value $b=y^{2}-x^{3} \bmod n$. If Lemma 2 is adopted, for the addition formula the sender $S$ must compute $a$ such that $a=\left(m_{y}^{2}-m_{x}^{3}\right) / m_{x} \bmod n$, and the receiver $\mathrm{R}$ must compute $a$ such that $a=\left(c_{y}^{2}-c_{x}^{3}\right) / c_{x} \bmod n$.

3. This scheme has the interesting property that it is not defined on a single group but on a large class of groups, all with the same order. The curve to be used is determined by the plaintext to be transmitted.

\section{Rabin-type Generalization}

\subsection{Protocol}

We propose another TOF (Type 2 Scheme) also based on elliptic curves over a ring, which is the Rabin-type generalization of the basic TOF (Type 1 scheme). The Type 2 scheme also overcomes the three deficiencies of the Type 0 scherne. For simplicity, we described the protocol for a public-key cryptosystem based on elliptic curves described in Lemma 1.

Step 0 (Key Generation) User U chooses large primes $p$ and $q$ such that

$$
p \equiv q \equiv 2(\bmod 3) \text {. }
$$

$\mathrm{U}$ computes the product $n=p q$, and the orders $N_{p}=\# E_{p}(0, b)=p+1$ and $N_{q}=\# E_{q}(0, b)=q+1$.

Summarizing, U's secret key is $p, q, N_{p}, N_{q}$, and U's public key is $n$. 
Step 1 (Encryption) A plaintext $M=\left(m_{x}, m_{y}\right)$ is an integer pair, where $m_{x} \in Z_{n}$, $m_{y} \in \mathrm{Z}_{n}$. Let $M=\left(m_{x}, m_{y}\right)$ be a point on the elliptic curve $E_{n}(0, b)$, where $b$ is determined by $m_{x}$ and $m_{y}$.

Sender A encrypts the point $M$ by doubling on the elliptic curve $E_{n}$ with the receiver's public key $n$ as

$$
C=2 \cdot M \text { over } E_{n}(0, b)
$$

and sends a ciphertext pair $C=\left(c_{x}, c_{y}\right)$ to a receiver $B$.

Step 2 (Decryption) Receiver B computes $M_{p} \in E_{p}(0, b)$ and $M_{q} \in E_{q}(0, b)$ from

$C_{p}=\left(c_{x} \bmod p, c_{y} \bmod p\right) \in E_{p}(0, b)$ and $C_{q}=\left(c_{x} \bmod q, c_{y} \bmod q\right) \in E_{q}(0, b)$ such that

$$
C_{p}=2 \cdot M_{p} \text { over } E_{p}(0, b), \quad C_{q}=2 \cdot M_{q} \text { over } E_{q}(0, b),
$$

by using a halving algorithm, which is described in Section 6.2.

B computes $M=\left(m_{x}, m_{y}\right) \in E_{n}$ from $M_{p}=\left(m_{p x}, m_{p y}\right) \in E_{p}(0, b)$ and $M_{q}=$ $\left(m_{q x}, m_{q y}\right) \in E_{q}(0, b)$ using the Chinese Remainder Theorem.

\section{[Notes]}

1. Since both $N_{p}$ and $N_{q}$ are even, 2 is not coprime to $N_{p}, N_{q}$ and $N_{n}$.

2. The Type 2 scheme has the drawback that there is $4: 1$ ambiguity in the decrypted messages, as is true for the original Rabin scheme.

3. In decryption based on a halving formula, the algorithm for finding a non-clouble point requires an exact expression of the elliptic curve. Thus, the receiver $B$ must compute $b$ such that $b=c_{y}^{2}-c_{x}^{3} \bmod n$.

\subsection{Halving Algorithm}

In general, points on $E_{p}(a, b): y^{2}=x^{3}+a x+b \bmod p$ can be separated into 2 classes, as integers in $\mathrm{Z}_{p}$ are classified into quadratic residue and quadratic non-residue modulo $p$.

Definition If $P=2 \cdot X$ over $E_{p}(a, b)$ for some point $X$ on the curve $E_{p}(a, b)$, then we call point $P$ a double point, where we denote the set of all double points by $D P_{p}$. If $P \neq 2 \cdot X$ over $E_{p}(a, b)$ for any point $X$, then we call point $P$ a non-double point, where we denote the set of all non-double points by $N D P_{n}$.

Double points and non-double points are distinguishable by using the following three lemmas, when the group structure of $E_{p}(a, b)$ is known.

Lemma 4. Assume that $E^{\prime}$ is a cyclic subgroup of $E_{p}(a, b)$ having the maximum order $N^{\prime}$. Let $P$ be in $E^{\prime}$, and $N^{\prime}$ be even. Then

$$
P \in D P_{p} \text { if and only if } N^{\prime} / 2 \cdot P=\mathcal{O} \text { over } E_{p}(a, b) \text {, }
$$

Lemma 5. Assume that $E^{\prime}$ is a cyclic subgroup of $E_{p}(a, b)$ with the maximum order $N^{\prime}$. Let $\alpha$ be the cardinality of $D P_{p}$ in $E^{\prime}$. Then 


$$
\alpha=\left\{\begin{aligned}
N^{\prime} / 2, & \text { if } N^{\prime} \text { is even; } \\
N^{\prime}, & \text { if } N^{\prime} \text { is odd. }
\end{aligned}\right.
$$

Lemma 6. Assume that $E_{p}(a, b)$ has the group structure $\mathrm{Z}_{(p+1) / 2} \times \mathrm{Z}_{2}$. Let $E^{\prime}$ be a cyclic subgroup of $E_{p}(a, b)$ with the maximum order $(p+1) / 2$ and let $Q$ be a point in this subgroup. Then

$P \in D P_{p}$ and $P \in E^{\prime}$ if and only if $e_{(p+1) / 2}(P, Q)=1$ and $(p+1) / 4 \cdot P=\mathcal{O}$ over $E_{p}(a, b)$, where $e_{(p+1) / 2}$ is the Weil pairing function $[8,19]$. Note that $(p+1) / 2$ is always even.

Next, consider a halving algorithm on elliptic curve $E_{p}(a, b)$ which outputs a half point of a given point over $E_{p}(a, b)$.

The algorithm of Adleman, Manders and Miller $[1,11]$ for computing a square root mod $p$ is easily adapted to a halving algorithm in $E_{p}(a, b)$. For completeness we describe the result.

Theorem 7. There exists an expected polynomial time algorithm which, given an odd prime $p$, an elliptic curve $E_{p}(a, b)$ in the case of Lemma 1 or $2, N_{p}$, and a point $Q \in D P_{p}$ as inputs, will output a half point of $Q$ over $E_{p}(a, b)$.

The proof of Theorem 7 is a direct consequence of the following algorithm.

Halving Algorithm on Elliptic Curve for Type 2 scheme

Input: $p$ (prime), $E_{p}(a, b), N_{p}, Q(=2 \cdot H) \in E_{p}(a, b)$.

Step 1. Compute an odd $c$, and $h$ such that $N_{p}=2^{h} c$.

Step 2. Choose random point $T$ such that $T \in N D P_{p}$ and $T$ is in the maximum cyclic subgroup including $Q$.

Step 3. Set $Y=Q, H=(c+1) / 2 \cdot Q$ over $E_{p}(a, b)$.

Step 4. Find the least $k$ such that $\left(2^{k} c\right) \cdot Y=\mathcal{O}$ over $E_{p}(a, b)$.

Step 5. If $k=0$ then output $I I$; else sct

$$
Y=Y-2^{h-k} \cdot T \text { over } E_{p}(a, b), \quad H=H-2^{h-k-1} \cdot c \cdot T \text { over } E_{p}(a, b)
$$

Output: $H$.

and go to step 4.

An algorithm for finding a non-double point $T$ is derived from Lemmas 4 and 6 as follows:

\section{Algorithm 1 for Finding a Non-Double Point $\left(E_{p}(a, b)\right.$ : cyclic)}

Input: $p$ (prime), $E_{p}(a, b), N_{p}$.

Step 1. Choose a random point $T=\left(t_{x}, t_{y}\right)$ on the curve.

Step 2. If $T$ is a non-double point, that is, $N_{p} / 2 \cdot T \neq \mathcal{O}$ over $E_{p}(a, b)$, then output $T$; else go to step 1 .

Output: $T=\left(t_{x}, t_{y}\right) \in N D P_{p}$.

Algorithm 2 for Finding a Non-Double Point $\left(E_{p}(a, 0) \cong \mathbf{Z}_{(p+1) / 2} \times Z_{2}\right)$

Input: $p$ (prime), $E_{p}(a, 0), N_{p}, Q \in E_{p}(a, 0)$.

Step 1. Choose a random point $T=\left(t_{x}, t_{y}\right)$ on the curve $E_{p}(a, 0)$ such that $e_{n}(Q, T)=1$.

Step 2. If $T$ is a non-double point, that is, $(p+1) / 4 \cdot T \neq \mathcal{O}$ over $E_{p}(a, 0)$, then output $T$; else go to stcp 1 . 
Output: $T$ such that $T=\left(t_{x}, t_{y}\right) \in N D P_{p}$, and $T \in E^{\prime}$, where $E^{\prime}$ is a cyclic subgroup of $E_{p}(a, 0)$ with the maximum orcier of $(p+1) / 2$ which includes point $Q$.

There exists a polynomial time general algorithm for finding a point on the elliptic curve [0]. In case 1 , for any $y \in Z_{p}$, the point $\left(\left(y^{2}-b\right)^{1 / 3}, y\right)$ is on the curve. Since $3 \not p-1$, the value of $\left(y^{2}-b\right)^{1 / 3}$ can be casily computed by $\left(y^{2}-b\right)^{\beta} \bmod p$, wherc $3 \beta \equiv 1$ $\bmod (p-1)$. In case 2 , for any $x \in Z_{p}$, the point $\left(x,\left(x^{3}+a x\right)^{1 / 2}\right)$ is on the curve. Since $p=4 k+3(k:$ integer $)$, the value of $\left(x^{3}+a x\right)^{1 / 2}$ can be easily computed by $\left(x^{3}+a x\right)^{k+1}$ $\bmod p$.

\section{Security}

The security of the proposed Type 1 scheme and Type 2 scheme over elliptic curves is based on the difficulty of factoring $n$. In this section, we discuss the security of these schemes from various viewpoints.

\subsection{Solving the Order}

The original RSA and Rabin schemes can be broken if one can determine order of the multiplicative groups. It is known that finding $\phi(n)=(p-1)(q-1)$ is computationally equivalent to factoring $n$. That is, the former is polynomially reducible to the latter, and vice versa. In our proposed schemes (Types 1 and 2 in the cases of Lemmas 1 and 2), a similar relationship holds.

Theorem 8. Let $N_{n}$ be $\operatorname{lcm}\left(\# E_{p}(a, b), \# E_{q}(a, b)\right)=\operatorname{lcm}(p+1, q+1)$. Finding $N_{n}$ is computationally equivalent to factoring the composite number $n$.

\subsection{Finding the Secret Key}

The security of the original RSA scheme is also based on the difficulty of finding the secret exponent key. The security of the Type 1 scheme is also based on the difficulty of finding the secret multiplier key $d$. We have the following relationship.

Theorem 9. Solving a secret key drom pablic keys $e$ and $n$ is computationally equivalent to factoring a composite number $n$.

\subsection{Complete Breaking}

Completely breaking Type 1 and 2 schemes means to recover both $m_{x}$ and $m_{y}$ from any ciphertext pair $\left(c_{x}, c_{y}\right)$ and the public keys. It is well known that completely breaking the original Rabin cryptosystem is as hard as factoring the composite $n$ used as the modulus. For the Type 2 scheme, we have the following theorem.

Theorem 10. Completely breaking the Type 2 scheme is computationally equivalent to factoring $n$.

Proof: It is clear that if once the factors of $n$ are known, plaintext $\left(m_{x}, m_{y}\right)$ can easily be computed from ciphertext $\left(c_{x}, c_{y}\right)$ and public keys $(a, n)$. Conversely, if there is an Algorithm $A 1$, given $P$ on $E_{n}(a, b)\left(E_{n}(0, b)\right.$ or $\left.E_{n}(a, 0)\right)$, to output $Q$ satisfying $P=$ $2 \cdot Q$ with non-negligible probablity, then we can construct an expected polynomial-time algorithm $\mathrm{B}$ to factor $n$, using $\mathrm{A} 1$ as an oracle. First, $\mathrm{B}$ chooses a random point $R=$ 
$\left(r_{x}, r_{y}\right)\left(r_{x}, r_{y} \in Z_{n}\right)$, and multiplies it by 2 , asks $A 1$ to halve this point, and B obtains $R^{\prime}$ satisfying $P=2 \cdot R^{\prime}$ with non-negligilole probablity. Then $\mathrm{B}$ computes $R_{0}=R-R^{\prime}$. Since $2 \cdot R_{0}=\mathcal{O}$, and $R_{0}$ over $E_{p}(a, b)\left(R_{0}\right.$ over $\left.E_{q}(a, b)\right)$ is $\mathcal{O}_{p}\left(\mathcal{O}_{q}\right)$, then $R_{0}$ is an undefined point with probability $1 / 2$. If $R_{0}$ is undefined, $\mathrm{B}$ can compute a non-trivial factor of $n$ by the extended Euclidean algorithm used for division modulo $n$. Clearly, the expected running time of $\mathrm{B}$ is polynomial-time in $\log n$.

In the Type 1 scheme, the equivalence between completely breaking this scheme and factoring $n$ is not known. This situation is the same as the original RSA scheme.

\subsection{Homomorphism Attacks and Their Countermeasures}

The encryption-decryption functions $\mathrm{E}(\cdot)$ and $\mathrm{D}(\cdot)$ for Type 1 and 2 schemes are homomorphic for addition as

$$
\mathrm{E}\left(M_{1}+M_{2}\right)=\mathrm{E}\left(M_{1}\right)+\mathrm{E}\left(M_{2}\right) \text { and } \mathrm{D}\left(M_{1}+M_{2}\right)=\mathrm{D}\left(M_{1}\right)+\mathrm{D}\left(M_{2}\right),
$$

for any points $M_{1}$ and $M_{2}$ on the same elliptic curve. This kind of homomorphic property is the basis for some attacking methods proposed [7] against the original RSA and Rabin schemes.

The probability that randomly chosen integer pairs $M_{1}$ and $M_{2}$ are on the same elliptic curve is as negligiblly small. Thus, passive attacks using homomorphism seem to be ineffective against Type 1 and 2 schemes.

Consider an active attack (a chosen-plaintext attack) using homomorphism. Suppose an attacker $A$ wants to make a victim $B$ sign a plaintext $M=\left(m_{x}, m_{y}\right)$ without $B$ 's consent. $A$ generates another message $M^{\prime}$ with $B^{\prime}$ s public keys $\left(e_{B}, n_{B}\right)$ and random integer $r$,

$$
M^{\prime}=M+e_{B} \cdot(r \cdot M) \text { over } E_{n_{B}},
$$

and sends $M^{\prime}$ to $B$. $B$ makes a signature $S^{\prime}$ for $M^{\prime}$ with his secret key $d_{B}$ :

$$
S^{\prime}=d_{B} \cdot M^{\prime}=d_{B} \cdot\left(M+e_{B} \cdot(r \cdot M)\right) \text { over } E_{n_{B}} .
$$

Then, $A$ computes a signature $S$ for $M$ from $S^{\prime}$ by

$$
S=S^{\prime}-r \cdot M \text { over } E_{n_{B}} .
$$

Using this technique, $A$ can forge $B$ 's signatures without $B$ 's secret key. To counter this attack, a randomization of a plaintext with a hashing function $h$ should be applied before the application of the function $\mathrm{D}$. This method is similar to that required for the original RSA scheme.

\subsection{Isomorphism Attacks and Their Countermeasures}

Definition Let $n=p q(p, q$ : prime $)$, and $E_{n}^{1}$ and $E_{n}^{2}$ be elliptic curves such that

$$
E_{n}^{1}: y^{2}=x^{3}+a_{1} x+b_{1} \bmod n, \quad E_{n}^{2}: y^{2}=x^{3}+a_{2} x+b_{2} \bmod n .
$$

$E_{n}^{1}$ and $E_{n}^{2}$ are isomorphic if there exist $u_{p} \in \mathrm{Z}_{p}^{*}$ and $u_{q} \in \mathbf{Z}_{q}^{*}$ such that

$$
a_{2} \equiv u_{p}^{4} a_{1} \bmod p, \quad b_{2} \equiv u_{p}^{6} b_{1} \bmod p
$$




$$
a_{2} \equiv u_{q}^{4} a_{1} \bmod q, \quad b_{2} \equiv u_{q}^{6} b_{1} \bmod q .
$$

Then the following isomorphic property of the elliptic curves over a ring is shown by using the property of the elliptic curves over a finite field and the Chinese- Remainder Theorem.

Lemma 11. Let $E_{n}^{1}$ and $E_{n}^{2}$ be elliptic curves such that

$$
E_{n}^{1}: y^{2}=x^{3}+a_{1} x+b_{1} \bmod n, \quad E_{n}^{2}: y^{2}=x^{3}+a_{2} x+b_{2} \bmod n .
$$

Let $M_{1}=\left(m_{1 x}, m_{1 y}\right), C_{1}=\left(c_{1 x}, c_{1 y}\right) \in E_{n}^{1}$ and $M_{2}=\left(m_{2 x}, m_{2 y}\right), C_{2}=\left(c_{2 x}, c_{2 y}\right) \in E_{n}^{2}$ where

$$
C_{1}=e \cdot M_{1} \text { over } E_{n}^{1}, \quad C_{2}=e \cdot M_{2} \text { over } E_{n}^{2} \text {. }
$$

Then the following statements are equivalent:

(i) $E_{n}^{1}$ and $E_{n}^{2}$ are isomorphic.

(ii) $a_{2} \equiv u^{4} a_{1} \bmod n, \quad b_{2} \equiv u^{6} b_{1} \bmod n \quad \exists u \in \mathrm{Z}_{n}^{*}$.

(iii) $c_{2 x} \equiv u^{2} c_{1 x} \bmod n, \quad c_{2 y} \equiv u^{3} c_{1 y} \bmod n \quad \exists u \in Z_{n}^{*}$.

(iv) $m_{2 x} \equiv u^{2} m_{1 x} \bmod n, \quad m_{2 y} \equiv u^{3} m_{1 y} \bmod n \quad \exists u \in Z_{n}^{*}$.

If $C_{1}, C_{2}$ and $M_{1}$ satisfying congruence (2) are given, then $M_{2}$ can be easily found by computing congruence (3). Notice that it is easy to check whether or not congruence (2) holds. If $M_{1}$ and $M_{2}$ are randomly chosen, then the probability that there exists $u$ satisfying congruence (2) is a negligibly small $1 / n$ for large $n$. Thus, passive attacks using isomorphism seem to be dificult for Types 1 and 2 schemes.

Consider an active attack (a chosen-plaintext attack) based on the isomorphic property of the elliptic curves. Suppose an attacker $A$ wants to make a victim $B$ sign a plaintext $M=\left(m_{x}, m_{y}\right)$ without $B^{\prime}$ s consent. $A$ generates another message $M I^{\prime}$ with $B$ 's public key $n_{B}$ and random integer $u$ :

$$
M^{\prime}=\left(u^{2} m_{x} \bmod n_{B}, u^{3} m_{y} \bmod n_{B}\right)
$$

and sends $M^{\prime}$ to $B$. $B$ makes a signature $S^{\prime}=\left(s_{x}^{\prime}, s_{y}^{\prime}\right)$ for $M^{\prime}$ with his secret key $d_{B}$ :

$$
S^{\prime}=d_{B} \cdot M^{\prime} \text { over } E_{n_{B}}^{\prime}
$$

Then, $A$ computes a signature $S=\left(s_{x}, s_{y}\right)$ for $M$ from $S^{\prime}$ by

$$
S=\left(s_{x}, s_{y}\right)=\left(u^{-2} s_{x}^{\prime} \bmod n_{B}, u^{-3} s_{y}^{\prime} \bmod n_{B}\right) .
$$

Note that the curve $E_{n_{B}}$ containing points $(M, S)$ and the curve $E_{n_{B}}^{\prime}$ containing points $\left(M^{\prime}, S^{\prime}\right)$ are isomorphic. Using this technique, $A$ can forge $B$ 's signatures without $B$ 's secret key. To counter this attack, the same technique described in Section 7.4 can be applied.

An attacker may try to forge a signature by using both homomorphism and isomorphism shown above. However, such combined attacks can also be prevented by randomization with the hash function $h$. 


\subsection{Security for Low Multiplier Attack}

Hastad [6] showed a low exponent attack on the original RSA and Rabin schemes when the same message is encrypted with several distinct moduli. He considered the problem of solving systems of congruences $P_{i}(m) \equiv 0\left(\bmod n_{i}\right) i=1, \ldots, k$, where $P_{i}$ are polynomial of degree $e$ and the $n_{i}$ are distinct relatively prime numbers and $m<\min n_{i}$. He proved that if $k>\frac{e(e+1)}{2}$, then $m$ can be recovered in polynomial time. Thus, he pointed out that enciphering linearly related messages with the RSA scheme with low exponent or the Rabin scheme is insecure. For the original RSA scheme, let $c=m^{e} \bmod n, c_{i}=m_{i}^{e}$ $\bmod n_{i}$, and $n=n_{1} \cdot n_{2} \cdots n_{k}$. In Hastad's algorithm, $c$ is first obtained from $c_{i}$ using the Chinese Remainder Theorem. Next, $m$ can be efficiently calculated from $c=m^{\text {e }}$ (with neglecting $n$ ), provided that $m^{e}<n$. For our proposed Types 1 and 2 schemes, let $C=e^{\prime} \cdot M$ over $E_{n}(a, b), C_{i}=e^{t} \cdot M$ over $E_{n_{i}}$, where $C=\left(c_{x}, c_{y}\right), M=\left(m_{x}, m_{y}\right)$, $C_{i}=\left(c_{i x}, c_{i y}\right)$. The value of $\left(c_{x}, c_{y}\right)$ is also obtained from $\left(c_{i x}, c_{i y}\right)$. However, it is difficult to solve $\left(m_{x}, m_{y}\right)$ from $\left(c_{x}, c_{y}\right)$ because $c_{x}$ and $c_{y}$ are expressed by rational equations in $m_{x}$ and $m_{y}$. Since the rational equations include divisions modulo $n$, if we transform the rational form relation into the polynomial form relation, the size of the coefficient of the polynomial form is of the order of n's size. Therefore, it seems impossible to solve the rational or polynomial form relation by neglecting modulus $n$. Thus, even if the multiplicr $e^{\prime}$ is small, a Hastad-like attack does not seem to work against the elliptic curve cryptosystems.

\section{Performance}

An elliptic curve addition $P_{1}+P_{2}$ on $E_{n}(a, b)$ requires one division, one squaring operation and one general multiplication in $\mathrm{Z}_{n}$ when $P_{1} \neq P_{2}$, and an extra squaring when $P_{1}=P_{2}$. (The much faster additions and subtractions in $Z_{n}$ are neglected for the sake of simplicity). Surprisingly, as opposed to $Z_{n}$ where squaring can be performed faster than a general multiplication, doubling a point on an elliptic curve is computationally more costly than adding two different points. This means that in order to compute a multiple $c \cdot P$ of a point $P$, an irregular addition chain for $c$ avoiding doubling operations should be used. When neglecting the fact that squaring in $Z_{n}$ can be implemented somewhat faster than a general multiplication, elliptic curve addition and doubling operations require about 2 and 3 multiplications in $Z_{n}$ and one division in $Z_{n}$, respectively.

Division in $\mathbf{Z}_{n}$ can be implemented by the generalized Euclidean algorithm for computing greatest common divisors. The most efficicnt algorithm for computing multiplicative inverses, however, is that invented by Massey [17], which is a gencralization of Stcin's algorithm [25]. However, a division in $Z_{n}$ seems to be less efficient than a multiplication in $\mathbf{Z}_{n}$.

On the other hand, if we calculate the addition on $E_{n}(a, b)$ in homogeneous coordinates, we can avoid the division in $\mathbf{Z}_{n}$ (except the final stage of the addition chain), although we must perform more multiplications.

Let $P_{1}=\left(x_{1}, y_{1}, z_{1}\right) \in E_{p}(a, b), P_{2}=\left(x_{2}, y_{2}, z_{2}\right) \in E_{p}(a, b)$, and suppose that $P_{1}, P_{2} \neq$ $\mathcal{O}, P_{1} \neq P_{2}$ and $P_{1} \neq-P_{2}$. The addition formula [9] for $E_{p}(a, b)$ to find $P_{3}=P_{1}+P_{2}=$ 
$\left(x_{3}, y_{3}, z_{3}\right)$ is given by

$$
\left\{\begin{array}{l}
x_{3}=v\left\{z_{2}\left(u^{2} z_{1}-2 v^{2} x_{1}\right)-v^{3}\right\} \bmod p \\
y_{3}=z_{2}\left(3 u v^{2} x_{1}-v^{3} y_{1}-u^{3} z_{1}\right)+u v^{3} \bmod p \\
z_{3}=v^{3} z_{1} z_{2} \bmod p
\end{array}\right.
$$

where $u=y_{2} z_{1}-y_{1} z_{2} \bmod p, v=x_{2} z_{1}-x_{1} z_{2} \bmod p$.

The doubling formula $[9]$ for $E_{p}(a, b)$ to find $P_{3}=2 \cdot P_{1}$ is given by

$$
\left\{\begin{array}{l}
x_{3}=2 y_{1} z_{1}\left(w^{2}-8 x_{1} y_{1}^{2} z_{1}\right) \bmod p \\
y_{3}=4 y_{1}^{2} z_{1}\left(3 w x_{1}-2 y_{1}^{2} z_{1}\right)-w^{3} \bmod p \\
z_{3}=8 y_{1}^{3} z_{1}^{3} \bmod p
\end{array}\right.
$$

where $w=3 x_{1}^{2}+a z_{1}^{2} \bmod p$.

One addition over $E_{n}(a, b)$ requires 12 multiplications in $\mathrm{Z}_{n}$, and one doubling over $E_{n}(a, b)$ requires 10 multiplications in $Z_{n}$, if $a=0$.

Therefore, in the affine coordinates, the computation required for our scheme (Scheme $1)$ is about $(2+c)$ times as much as that for the RSA scheme, where $c$ is the ratio of the computation amount of division in $Z_{n}$ to that of multiplication in $Z_{n}$. On the other hand, in the homogeneous coordinates, the computation required for encryption with our scheme is about 11 times as much as that for the RSA scheme. Since in our elliptic curve system a message consists of two elements of $Z_{n}$ compared to only one in the RSA system, the computation speed of our scheme is about $2 /(2+c)$ or $1 / 6$ of the speed of RSA.

\section{Conclusions}

We have proposed new public key cryptosystems based on elliptic curves modulo $n$, where $n$ is a product of two large primes. Furthermore, we have given some analysis of the security of these systems. For the proposed Type 1 scheme, the master key concept [10] and the blind signature concept [2] are similarly applicable (using the combined techniques of Sections 7.4 and 7.5).

\section{Acknowledgements}

We would like to thank Martin Benninger, Burt Kaliski, Neal Koblitz, Alfred Menezes, Pierre Schmid, and Hiroki Shizuya for helpful discussions and for their support.

\section{References}

[1] L. Adleman, K. Manders, and G. Miller, "On taking roots in finite fields", 20lh FOCS, Vol. 20, 1977, pp.175-178 (1977).

[2] D. Chaum, "Blind signatures for untraceable payments", Proc. of Crypto'82, pp.199-203 (1982). 
[3] W. Diffie and M.E. Hellman, "New directions in cryptography, IEEE Transactions on Information Theory," Vol. 22, No. 6, pp. 644-654 (1976).

[4] T. El-Gamal, "A public key cryptosystem and a signature scheme based on the discrete logarithm", IEEE Transactions on Information Theory, Vol. 31, No. 4, pp. 469-472 (1985).

[5] S. Goldwasser and J. Kilian, "Almost all primes can be quickly certified", Proc. of the 18th Annual ACM Symposium on the Theory of Computing, pp. 316-320 (1986).

[6] J. Hastad, "On using RSA with low exponent in a public key network", Proc. of Crypto'85, pp.403-408 (1985).

[7] W. Jonge and D. Chaum, “Attacks on some RSA signatures”, Proc. of Crypto's5, pp.18-27 (1985).

[8] B.S. Kaliski, "A pseudo-random bit generator based on elliptic logarithms", Proc. of Crypto'86, pp. 84-103 (1987).

[9] N. Koblitz, A Course in Number Theory and Cryptogmphy, Berlin: Springer-Verlag, (1987).

[10] K. Koyama, "A Master Key for the RSA public-key cryptosystem", I.E.C.E. Trans.(D), J-65, 2, pp.163-170 (1982).

[11] E. Kranakis, Primality and Cryptogmphy, Stuttgart: Teubner, and New York: John Wiley \& Sons, (1986).

[12] A.K. Lenstra, H.W. Lenstra, M.S. Manasse and J.M. Pollard, "The number field sieve", Proc. of STOC'90, pp.564-572 (1990).

[13] A.K. Lenstra and M.S. Manasse, "Factoring with two large primes", Proc. of EUROCRYPT'00, pp.72-82 (1991)

[14] H.W. Lenstra, "Factoring integers with elliptic curves", Annals of Mathematics, Vol. 126, pp. 649-673 (1987).

[15] U.M. Maurer, "Fast generation of RSA-moduli with almost maximal diversity", Proc. of EUROCRYPT'89, pp. 636-647 (1990).

[16] U.M. Maurer and Y. Yacobi, "Non-interactive public-key cryptography", to appear in Proc. Eurocrypt '91.

[17] J.L. Massey, "Cryptography - fundamentals and applications (Copies of transparencies)", Advanced Technology Seminars, Zurich, Switzerland, (1988).

[18] G.L. Miller, "Riemann's hypothesis and tests for primality", J. Comput. System Sci. Vol.13, pp.300-317, (1976).

[19] A.J. Menezes, T. Okamoto, S.A. Vanstone, "Reducing Elliptic Curve Logarithms to Logarithms in a Finite Field", Proc. of STOC'91, pp.80-×y (1991).

[20] M.O. Rabin, "Probabilistic algorithm for testing primality", Journal on Number Theory, Vol. 12, pp. 128-138 (1980).

[21] R.L. Rivest, A. Shamir, and L. Adleman, " $\Lambda$ method for obtaining digital signatures and public-key cryptosystems", Communications of the ACM, Vol. 21, No. 2, pp. 120-126 (1978). 
[22] R. Schoof, "Elliptic curves over finite fields and the computation of square roots mod $p$ ", Mathematics of Computation, Vol. 44, No. 170, pp. 483-494 (1985).

[23] J.H. Silverman, The Arithmetic of Elliptic Curves, Springer-Verlag, (1986).

[24] G. Simmons and M. Norris, "Preliminary comments on the M.I.T public key cryptosystem", Cryptologia, Vol. 1, No. 4, pp. 406-414 (1977).

[25] J. Stein, "Computational problems associated with Racah algebra", J. Comp. Plys., Vol. 1, pp. 397-405 (1961). 